\title{
Pre-training is a Hot Topic: Contextualized Document Embeddings Improve Topic Coherence
}

\author{
Federico Bianchi \\ Bocconi University \\ Via Sarfatti 25, 20136 \\ Milan, Italy \\ f.bianchi@unibocconi.it
}

\author{
Silvia Terragni \\ University of Milan-Bicocca \\ Viale Sarca 336, 20126 \\ Milan, Italy \\ s.terragni4@campus.unimib.it
}

\author{
Dirk Hovy \\ Bocconi University \\ Via Sarfatti 25, 20136 \\ Milan, Italy \\ dirk.hovy@unibocconi.it
}

\begin{abstract}
Topic models extract groups of words from documents, whose interpretation as a topic hopefully allows for a better understanding of the data. However, the resulting word groups are often not coherent, making them harder to interpret. Recently, neural topic models have shown improvements in overall coherence. Concurrently, contextual embeddings have advanced the state of the art of neural models in general. In this paper, we combine contextualized representations with neural topic models. We find that our approach produces more meaningful and coherent topics than traditional bag-of-words topic models and recent neural models. Our results indicate that future improvements in language models will translate into better topic models.
\end{abstract}

\section{Introduction}

One of the crucial issues with topic models is the quality of the topics they discover. Coherent topics are easier to interpret and are considered more meaningful. E.g., a topic represented by the words "apple, pear, lemon, banana, kiwi" would be considered a meaningful topic on FRUIT and is more coherent than one defined by "apple, knife, lemon, banana, spoon." Coherence can be measured in numerous ways, from human evaluation via intrusion tests (Chang et al., 2009) to approximated scores (Lau et al., 2014; Röder et al., 2015).

However, most topic models still use Bag-ofWords (BoW) document representations as input. These representations, though, disregard the syntactic and semantic relationships among the words in a document, the two main linguistic avenues to coherent text. I.e., BoW models represent the input in an inherently incoherent manner.

Meanwhile, pre-trained language models are becoming ubiquitous in Natural Language Processing (NLP), precisely for their ability to cap- ture and maintain sentential coherence. Bidirectional Encoder Representations from Transformers (BERT) (Devlin et al., 2019), the most prominent architecture in this category, allows us to extract pre-trained word and sentence representations. Their use as input has advanced state-of-the-art performance across many tasks. Consequently, BERT representations are used in a diverse set of NLP applications (Rogers et al., 2020; Nozza et al., 2020).

Various extensions of topic models incorporate several types of information (Xun et al., 2017; Zhao et al., 2017; Terragni et al., 2020a), use word relationships derived from external knowledge bases (Chen et al., 2013; Yang et al., 2015; Terragni et al., 2020b), or pre-trained word embeddings (Das et al., 2015; Dieng et al., 2020; Nguyen et al., 2015; Zhao et al., 2017). Even for neural topic models, there exists work on incorporating external knowledge, e.g., via word embeddings (Gupta et al., 2019, 2020; Dieng et al., 2020).

In this paper, we show that adding contextual information to neural topic models provides a significant increase in topic coherence. This effect is even more remarkable given that we cannot embed long documents due to the sentence length limit in recent pre-trained language models architectures.

Concretely, we extend Neural ProdLDA (Product-of-Experts LDA) (Srivastava and Sutton, 2017), a state-of-the-art topic model that implements black-box variational inference (Ranganath et al., 2014), to include contextualized representations. Our approach leads to consistent and significant improvements in two standard metrics on topic coherence and produces competitive topic diversity results.

Contributions We propose a straightforward and easily implementable method that allows neural topic models to create coherent topics. We show 
that the use of contextualized document embeddings in neural topic models produces significantly more coherent topics. Our results suggest that topic models benefit from latent contextual information, which is missing in BoW representations. The resulting model addresses one of the most central issues in topic modeling. We release our implementation as a Python library, available at the following link: https://github.com/MilaNLProc/ contextualized-topic-models.

\section{Neural Topic Models with Language Model Pre-training}

We introduce a Combined Topic Model (CombinedTM) to investigate the incorporation of contextualized representations in topic models. Our model is built around two main components: (i) the neural topic model ProdLDA (Srivastava and Sutton, 2017) and (ii) the SBERT embedded representations (Reimers and Gurevych, 2019). Let us notice that our method is indeed agnostic about the choice of the topic model and the pre-trained representations, as long as the topic model extends an autoencoder and the pre-trained representations embed the documents.

ProdLDA is a neural topic modeling approach based on the Variational AutoEncoder (VAE). The neural variational framework trains a neural inference network to directly map the BoW document representation into a continuous latent representation. Then, a decoder network reconstructs the BoW by generating its words from the latent document representation ${ }^{1}$. The framework explicitly approximates the Dirichlet prior using Gaussian distributions, instead of using a Gaussian prior like Neural Variational Document Models (Miao et al., 2016). Moreover, ProdLDA replaces the multinomial distribution over individual words in LDA with a product of experts (Hinton, 2002).

We extend this model with contextualized document embeddings from SBERT (Reimers and Gurevych, 2019), ${ }^{2}$ a recent extension of BERT that allows the quick generation of sentence embeddings. This approach has one limitation. If a document is longer than SBERT's sentence-length limit, the rest of the document will be lost. The document representations are projected through a hidden layer with the same dimensionality as the vocabulary size, concatenated with the BoW repre-

\footnotetext{
${ }^{1}$ For more details see (Srivastava and Sutton, 2017).

${ }^{2}$ https://github.com/UKPLab/ sentence-transformers
}

sentation. Figure 1 briefly sketches the architecture of our model. The hidden layer size could be tuned, but an extensive evaluation of different architectures is out of the scope of this paper.

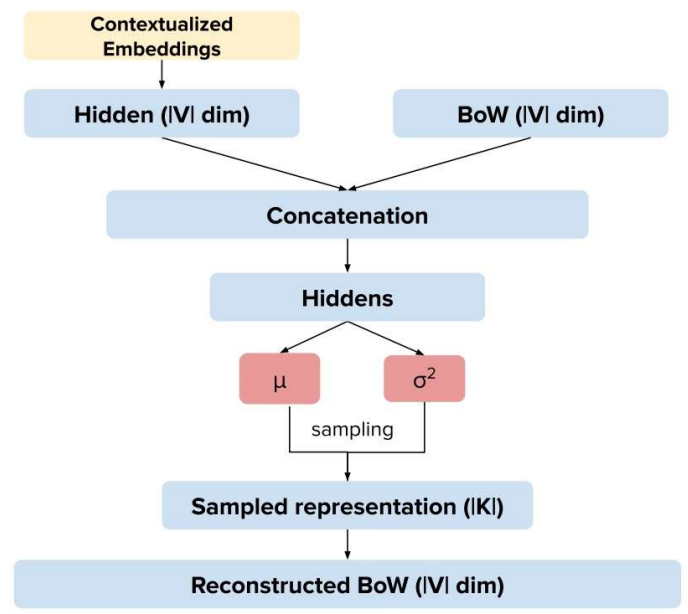

Figure 1: High-level sketch of CombinedTM. Refer to (Srivastava and Sutton, 2017) for more details on the architecture we extend.

\begin{tabular}{l|rr}
\hline Dataset & Docs & Vocabulary \\
\hline 20Newsgroups & 18,173 & 2,000 \\
Wiki20K & 20,000 & 2,000 \\
StackOverflow & 16,408 & 2,303 \\
Tweets2011 & 2,471 & 5,098 \\
Google News & 11,108 & 8,110 \\
\hline
\end{tabular}

Table 1: Statistics of the datasets used.

\section{Experimental Setting}

We provide detailed explanations of the experiments (e.g., runtimes) in the supplementary materials. To reach full replicability, we use open-source implementations of the algorithms.

\subsection{Datasets}

We evaluate the models on five datasets: 20NewsGroups $^{3}$, Wiki20K (a collection of 20,000 English Wikipedia abstracts from Bianchi et al. (2021)), Tweets $2011^{4}$, Google News (Qiang et al., 2019), and the StackOverflow dataset (Qiang et al., 2019). The latter three are already pre-processed. We use a similar pipeline for 20NewsGroups and Wiki20K: removing digits, punctuation, stopwords, and infrequent words. We derive SBERT document representations from unpreprocessed text for Wiki20k

\footnotetext{
${ }^{3}$ http: //qwone.com/ jason/20Newsgroups/

${ }^{4}$ https://trec.nist.gov/data/tweets/
} 


\begin{tabular}{|c|c|c|c|}
\hline Model & $\operatorname{Avg} \tau$ & $\operatorname{Avg} \alpha$ & $\operatorname{Avg} \rho$ \\
\hline \multicolumn{4}{|c|}{ Results for the Wiki20K Dataset: } \\
\hline Ours & 0.1823 & 0.1980 & 0.9950 \\
\hline PLDA & 0.1397 & 0.1799 & 0.9901 \\
\hline MLDA & 0.1443 & 0.2110 & 0.9843 \\
\hline NVDM & -0.2938 & 0.0797 & 0.9604 \\
\hline ETM & 0.0740 & 0.1948 & 0.8632 \\
\hline LDA & -0.0481 & 0.1333 & 0.9931 \\
\hline \multicolumn{4}{|c|}{ Results for the StackOverflow Dataset: } \\
\hline Ours & 0.0280 & 0.1563 & 0.9805 \\
\hline PLDA & -0.0394 & 0.1370 & 0.9914 \\
\hline MLDA & 0.0136 & 0.1450 & 0.9822 \\
\hline NVDM & -0.4836 & 0.0985 & 0.8903 \\
\hline ETM & -0.4132 & 0.1598 & 0.4788 \\
\hline LDA & -0.3207 & 0.1063 & 0.8947 \\
\hline \multicolumn{4}{|c|}{ Results for the GoogleNews Dataset: } \\
\hline Ours & 0.1207 & 0.1325 & 0.9965 \\
\hline PLDA & 0.0110 & 0.1218 & 0.9902 \\
\hline MLDA & 0.0849 & 0.1219 & 0.9959 \\
\hline NVDM & -0.3767 & 0.1067 & 0.9648 \\
\hline ETM & -0.2770 & 0.1175 & 0.4700 \\
\hline LDA & -0.3250 & 0.0969 & 0.9774 \\
\hline \multicolumn{4}{|c|}{ Results for the Tweets2011 Dataset: } \\
\hline Ours & 0.1008 & 0.1493 & 0.9901 \\
\hline PLDA & 0.0612 & 0.1327 & 0.9847 \\
\hline MLDA & 0.0122 & 0.1272 & 0.9956 \\
\hline NVDM & -0.5105 & 0.0797 & 0.9751 \\
\hline ETM & -0.3613 & 0.1166 & 0.4335 \\
\hline LDA & -0.3227 & 0.1025 & 0.8169 \\
\hline \multicolumn{4}{|c|}{ Results for the 20NewsGroups Dataset: } \\
\hline Ours & 0.1025 & 0.1715 & 0.9917 \\
\hline PLDA & 0.0632 & 0.1554 & 0.9931 \\
\hline MLDA & 0.1300 & 0.2210 & 0.9808 \\
\hline NVDM & -0.1720 & 0.0839 & 0.9805 \\
\hline ETM & 0.0766 & 0.2539 & 0.8642 \\
\hline LDA & 0.0173 & 0.1627 & 0.9897 \\
\hline
\end{tabular}

Table 2: Averaged results over 5 numbers of topics. Best results are marked in bold.

and 20NewsGroups. For the others, we use the pre-processed text; ${ }^{5}$ See Table 1 for dataset statistics. The sentence encoding model used is the pretrained RoBERTa model fine-tuned on SNLI (Bowman et al., 2015), MNLI (Williams et al., 2018),

\footnotetext{
${ }^{5}$ This can be sub-optimal, but many datasets in the literature are already pre-processed.
}

and the STSb (Cer et al., 2017) dataset. ${ }^{6}$

\subsection{Metrics}

We evaluate each model on three different metrics: two for topic coherence (normalized pointwise mutual information and a word-embedding based measure) and one metric to quantify the diversity of the topic solutions.

Normalized Pointwise Mutual Information ( $\tau$ ) (Lau et al., 2014) measures how related the top-10 words of a topic are to each other, considering the words' empirical frequency in the original corpus. $\tau$ is a symbolic metric and relies on co-occurrence. As Ding et al. (2018) pointed out, though, topic coherence computed on the original data is inherently limited. Coherence computed on an external corpus, on the other hand, correlates much more to human judgment, but it may be expensive to estimate.

External word embeddings topic coherence $(\alpha)$ provides an additional measure of how similar the words in a topic are. We follow Ding et al. (2018) and first compute the average pairwise cosine similarity of the word embeddings of the top-10 words in a topic, using Mikolov et al. (2013) embeddings. Then, we compute the overall average of those values for all the topics. We can consider this measure as an external topic coherence, but it is more efficient to compute than Normalized Pointwise Mutual Information on an external corpus.

Inversed Rank-Biased Overlap ( $\rho$ ) evaluates how diverse the topics generated by a single model are. We define $\rho$ as the reciprocal of the standard RBO (Webber et al., 2010; Terragni et al., 2021b). RBO compares the 10-top words of two topics. It allows disjointedness between the lists of topics (i.e., two topics can have different words in them) and uses weighted ranking. I.e., two lists that share some of the same words, albeit at different rankings, are penalized less than two lists that share the same words at the highest ranks. $\rho$ is 0 for identical topics and 1 for completely different topics.

\subsection{Models}

Our main objective is to show that contextual information increases coherence. To show this, we compare our approach to ProdLDA (Srivastava and Sutton, 2017, the model we extend) ${ }^{7}$, and the

\footnotetext{
${ }^{6}$ stsb-roberta-large

${ }^{7}$ We use the implementation of Carrow (2018).
} 
following models: (ii) Neural Variational Document Model (NVDM) (Miao et al., 2016); (iii) the very recent ETM (Dieng et al., 2020), MetaLDA (MLDA) (Zhao et al., 2017) and (iv) LDA (Blei et al., 2003).

\subsection{Configurations}

To maximize comparability, we train all models with similar hyper-parameter configurations. The inference network for both our method and ProdLDA consists of one hidden layer and 100dimension of softplus units, which converts the input into embeddings. This final representation is again passed through a hidden layer before the variational inference process. We follow (Srivastava and Sutton, 2017) for the choice of the parameters. The priors over the topic and document distributions are learnable parameters. For LDA, the Dirichlet priors are estimated via ExpectationMaximization. See the Supplementary Materials for additional details on the configurations.

\section{Results}

We divide our results into two parts: we first describe the results for our quantitative evaluation, and we then explore the effect on the performance when we use two different contextualized representations.

\subsection{Quantitative Evaluation}

We compute all the metrics for $25,50,75,100$, and 150 topics. We average results for each metric over 30 runs of each model (see Table 2).

As a general remark, our model provides the most coherent topics across all corpora and topic settings, even maintaining a competitive diversity of the topics. This result suggests that the incorporation of contextualized representations can improve a topic model's performance.

LDA and NVDM obtain low coherence. This result has also also been confirmed by Srivastava and Sutton (2017). ETM shows good external coherence $(\alpha)$, especially in 20NewsGroups and StackOverflow. However, it fails at obtaining a $\operatorname{good} \tau$ coherence for short texts. Moreover, $\rho$ shows that the topics are very similar to one another. A manual inspection of the topics confirmed this problem. MetaLDA is the most competitive of the models we used for comparison. This may be due to the incorporation of pre-trained word embeddings into MetaLDA. Our model provides very competitive results, and the second strongest model appears to be

\begin{tabular}{|c|c|c|c|c|c|}
\hline Wiki20K & 25 & 50 & 75 & 100 & 150 \\
\hline Ours & $0.17^{\star}$ & $0.19^{*}$ & $0.18^{*}$ & $0.19^{*}$ & $0.17^{*}$ \\
\hline MLDA & 0.15 & 0.15 & 0.14 & 0.14 & 0.13 \\
\hline \multicolumn{6}{|l|}{ SO } \\
\hline Ours & 0.05 & $0.03^{*}$ & $0.02 *$ & $0.02^{*}$ & $0.02 *$ \\
\hline MLDA & $0.05^{+}$ & 0.02 & 0.00 & -0.02 & 0.00 \\
\hline \multicolumn{6}{|l|}{ GNEWS } \\
\hline Ours & $-0.03^{*}$ & $0.10^{*}$ & $0.15^{*}$ & $0.18^{*}$ & $0.19^{*}$ \\
\hline MLDA & -0.06 & 0.07 & 0.13 & 0.16 & 0.14 \\
\hline \multicolumn{6}{|l|}{ Tweets } \\
\hline Ours & $0.05^{+}$ & $0.10^{*}$ & $0.11^{*}$ & $0.12^{*}$ & $0.12^{*}$ \\
\hline MLDA & 0.00 & 0.05 & 0.06 & 0.04 & -0.07 \\
\hline \multicolumn{6}{|l|}{ 20NG } \\
\hline Ours & 0.12 & 0.11 & 0.10 & 0.09 & 0.09 \\
\hline MLDA & $0.13^{*}$ & $0.13^{*}$ & $0.13^{*}$ & $0.13^{*}$ & $0.12^{*}$ \\
\hline
\end{tabular}

Table 3: Comparison of $\tau$ between CombinedTM (ours) and MetaLDA over various choices of topics. Each result averaged over 30 runs. ${ }^{*}$ indicates statistical significance of the results (t-test, $\mathrm{p}$-value $<0.05$ ).

MetaLDA. For this reason, we provide a detailed comparison of $\tau$ in Table 3, where we show the average coherence for each number of topics; we show that on 4 datasets over 5 our model provides the best results, but still keeping a very competitive score on 20NG, where MetaLDA is best.

Readers can see examples of the top words for each model in the Supplementary Materials. These descriptors illustrate the increased coherence of topics obtained with SBERT embeddings.

\subsection{Using Different Contextualized Representations}

Contextualized representations can be generated from different models and some representations might be better than others. Indeed, one question left to answer is the impact of the specific contextualized model on the topic modeling task. To answer to this question we rerun all the experiments with CombinedTM but we used different contextualized sentence embedding methods as input to the model.

We compare the performance of CombinedTM using two different models for embedding the contextualized representations found in the SBERT repository: ${ }^{8}$ stsb-roberta-large (Ours-R), as employed in the previous experimental setting, and using bert-base-nli-means (Ours-B). The latter is derived from a BERT model fine-tuned on NLI

\footnotetext{
${ }^{8}$ https://github.com/UKPLab/ sentence-transformers
} 


\begin{tabular}{cccccc}
\hline & Wiki20K & SO & GN & Tweets & 20NG \\
\hline Ours-R & $\mathbf{0 . 1 8}$ & $\mathbf{0 . 0 3}$ & $\mathbf{0 . 1 2}$ & $\mathbf{0 . 1 0}$ & $\mathbf{0 . 1 0}$ \\
Ours-B & $\mathbf{0 . 1 8}$ & 0.02 & 0.08 & 0.06 & 0.07 \\
\hline
\end{tabular}

Table 4: $\tau$ performance of CombinedTM using different contextualized encoders.

data. Table 4 shows the coherence of the two approaches on all the datasets (we averaged all results). In these experiments, RoBERTa fine-tuned on the STSb dataset has a strong impact on the increase of the coherence. This result suggests that including novel and better contextualized embeddings can further improve a topic model's performance.

\section{Related Work}

In recent years, neural topic models have gained increasing success and interest (Zhao et al., 2021; Terragni et al., 2021a), due to their flexibility and scalability. Several topic models use neural networks (Larochelle and Lauly, 2012; Salakhutdinov and Hinton, 2009; Gupta et al., 2020) or neural variational inference (Miao et al., 2016; Mnih and Gregor, 2014; Srivastava and Sutton, 2017; Miao et al., 2017; Ding et al., 2018). Miao et al. (2016) propose NVDM, an unsupervised generative model based on VAEs, assuming a Gaussian distribution over topics. Building upon NVDM, Dieng et al. (2020) represent words and topics in the same embedding space. Srivastava and Sutton (2017) propose a neural variational framework that explicitly approximates the Dirichlet prior using a Gaussian distribution. Our approach builds on this work but includes a crucial component, i.e., the representations from a pre-trained transformer that can benefit from both general language knowledge and corpusdependent information. Similarly, Bianchi et al. (2021) replace the BOW document representation with pre-trained contextualized representations to tackle a problem of cross-lingual zero-shot topic modeling. This approach was extended by Mueller and Dredze (2021) that also considered fine-tuning the representations. A very recent approach (Hoyle et al., 2020) which follows a similar direction uses knowledge distillation (Hinton et al., 2015) to combine neural topic models and pre-trained transformers.

\section{Conclusions}

We propose a straightforward and simple method to incorporate contextualized embeddings into topic models. The proposed model significantly improves the quality of the discovered topics. Our results show that context information is a significant element to consider also for topic modeling.

\section{Ethical Statement}

In this research work, we used datasets from the recent literature, and we do not use or infer any sensible information. The risk of possible abuse of our models is low.

\section{Acknowledgments}

We thank our colleague Debora Nozza and Wray Buntine for providing insightful comments on a previous version of this paper. Federico Bianchi and Dirk Hovy are members of the Bocconi Institute for Data Science and Analytics (BIDSA) and the Data and Marketing Insights (DMI) unit.

\section{References}

Federico Bianchi, Silvia Terragni, Dirk Hovy, Debora Nozza, and Elisabetta Fersini. 2021. Cross-lingual contextualized topic models with zero-shot learning. In Proceedings of the 16th Conference of the European Chapter of the Association for Computational Linguistics: Main Volume, pages 1676-1683, Online. Association for Computational Linguistics.

David M. Blei, Andrew Y. Ng, and Michael I. Jordan. 2003. Latent dirichlet allocation. J. Mach. Learn. Res., 3:993-1022.

Samuel R. Bowman, Gabor Angeli, Christopher Potts, and Christopher D. Manning. 2015. A large annotated corpus for learning natural language inference. In Proceedings of the 2015 Conference on Empirical Methods in Natural Language Processing, pages 632-642, Lisbon, Portugal. Association for Computational Linguistics.

Stephen Carrow. 2018. PyTorchAVITM: Open Source AVITM Implementation in PyTorch. Github.

Daniel Cer, Mona Diab, Eneko Agirre, Iñigo LopezGazpio, and Lucia Specia. 2017. SemEval-2017 task 1: Semantic textual similarity multilingual and crosslingual focused evaluation. In Proceedings of the 11th International Workshop on Semantic Evaluation (SemEval-2017), pages 1-14, Vancouver, Canada. Association for Computational Linguistics.

Jonathan Chang, Jordan L. Boyd-Graber, Sean Gerrish, Chong Wang, and David M. Blei. 2009. Reading tea leaves: How humans interpret topic models. In Advances in Neural Information Processing Systems 22: 23 rd Annual Conference on Neural Information Processing Systems 2009, pages 288-296. Curran Associates, Inc. 
Zhiyuan Chen, Arjun Mukherjee, Bing Liu, Meichun Hsu, Malú Castellanos, and Riddhiman Ghosh. 2013. Discovering coherent topics using general knowledge. In 22nd ACM International Conference on Information and Knowledge Management, CIKM'13, San Francisco, CA, USA, October 27. November 1, 2013, pages 209-218. ACM.

Rajarshi Das, Manzil Zaheer, and Chris Dyer. 2015. Gaussian LDA for topic models with word embeddings. In Proceedings of the 53rd Annual Meeting of the Association for Computational Linguistics and the 7th International Joint Conference on Natural Language Processing of the Asian Federation of Natural Language Processing, ACL 2015, pages 795804. The Association for Computational Linguistics.

Jacob Devlin, Ming-Wei Chang, Kenton Lee, and Kristina Toutanova. 2019. BERT: pre-training of deep bidirectional transformers for language understanding. In Proceedings of the 2019 Conference of the North American Chapter of the Association for Computational Linguistics: Human Language Technologies, NAACL-HLT 2019, pages 4171-4186.

Adji B. Dieng, Francisco J. R. Ruiz, and David M. Blei. 2020. Topic modeling in embedding spaces. Transactions of the Association for Computational Linguistics, 8:439-453.

Ran Ding, Ramesh Nallapati, and Bing Xiang. 2018. Coherence-aware neural topic modeling. In Proceedings of the 2018 Conference on Empirical Methods in Natural Language Processing, Brussels, Belgium, October 31 - November 4, 2018, pages 830836.

Pankaj Gupta, Yatin Chaudhary, Florian Buettner, and Hinrich Schütze. 2019. Document informed neural autoregressive topic models with distributional prior. In $A A A I 2019$, pages 6505-6512. AAAI Press.

Pankaj Gupta, Yatin Chaudhary, Thomas Runkler, and Hinrich Schuetze. 2020. Neural topic modeling with continual lifelong learning. In International Conference on Machine Learning, pages 3907-3917. PMLR.

Geoffrey Hinton, Oriol Vinyals, and Jeffrey Dean. 2015. Distilling the knowledge in a neural network. In NIPS Deep Learning and Representation Learning Workshop.

Geoffrey E. Hinton. 2002. Training products of experts by minimizing contrastive divergence. Neural Computation, 14(8):1771-1800.

Alexander Miserlis Hoyle, Pranav Goel, and Philip Resnik. 2020. Improving Neural Topic Models using Knowledge Distillation. In Proceedings of the 2020 Conference on Empirical Methods in Natural Language Processing (EMNLP), pages 1752-1771, Online. Association for Computational Linguistics.
Hugo Larochelle and Stanislas Lauly. 2012. A neural autoregressive topic model. In Advances in Neural Information Processing Systems 25: 26th Annual Conference on Neural Information Processing Systems 2012, pages 2717-2725.

Jey Han Lau, David Newman, and Timothy Baldwin. 2014. Machine reading tea leaves: Automatically evaluating topic coherence and topic model quality. In Proceedings of the 14th Conference of the European Chapter of the Association for Computational Linguistics, EACL 2014, pages 530-539.

Yishu Miao, Edward Grefenstette, and Phil Blunsom. 2017. Discovering discrete latent topics with neural variational inference. In Proceedings of the 34th International Conference on Machine Learning, ICML 2017, volume 70 of Proceedings of Machine Learning Research, pages 2410-2419. PMLR.

Yishu Miao, Lei Yu, and Phil Blunsom. 2016. Neural variational inference for text processing. In Proceedings of the 33nd International Conference on Machine Learning, ICML 2016, New York City, NY, USA, June 19-24, 2016, volume 48 of JMLR Workshop and Conference Proceedings, pages 1727 1736. JMLR.org.

Tomas Mikolov, Ilya Sutskever, Kai Chen, Greg S Corrado, and Jeff Dean. 2013. Distributed representations of words and phrases and their compositionality. In Advances in Neural Information Processing Systems 26, pages 3111-3119. Curran Associates, Inc.

Andriy Mnih and Karol Gregor. 2014. Neural variational inference and learning in belief networks. In Proceedings of the 31th International Conference on Machine Learning, ICML 2014, volume 32 of JMLR Workshop and Conference Proceedings, pages 17911799. JMLR.org.

Aaron Mueller and Mark Dredze. 2021. Fine-tuning encoders for improved monolingual and zero-shot polylingual neural topic modeling. In Proceedings of the 2021 Conference of the North American Chapter of the Association for Computational Linguistics: Human Language Technologies, pages 3054-3068, Online. Association for Computational Linguistics.

Dat Quoc Nguyen, Richard Billingsley, Lan Du, and Mark Johnson. 2015. Improving topic models with latent feature word representations. Transactions of the Association for Computational Linguistics, 3:299-313.

Debora Nozza, Federico Bianchi, and Dirk Hovy. 2020. What the [MASK]? Making Sense of Language-Specific BERT Models. arXiv preprint arXiv:2003.02912.

Jipeng Qiang, Qian Zhenyu, Yun Li, Yunhao Yuan, and Xindong Wu. 2019. Short text topic modeling techniques, applications, and performance: A survey. arXiv preprint arXiv:1904.07695. 
Rajesh Ranganath, Sean Gerrish, and David M. Blei. 2014. Black box variational inference. In Proceedings of the Seventeenth International Conference on Artificial Intelligence and Statistics, AISTATS 2014, 2014, volume 33 of JMLR Workshop and Conference Proceedings, pages 814-822. JMLR.org.

Nils Reimers and Iryna Gurevych. 2019. SentenceBERT: Sentence embeddings using Siamese BERTnetworks. In Proceedings of the 2019 Conference on Empirical Methods in Natural Language Processing and the 9th International Joint Conference on Natural Language Processing (EMNLP-IJCNLP), pages 3982-3992, Hong Kong, China. Association for Computational Linguistics.

Michael Röder, Andreas Both, and Alexander Hinneburg. 2015. Exploring the space of topic coherence measures. In Proceedings of the Eighth ACM International Conference on Web Search and Data Mining, WSDM 2015, pages 399-408. ACM.

Anna Rogers, Olga Kovaleva, and Anna Rumshisky. 2020. A primer in BERTology: What we know about how BERT works. Transactions of the Association for Computational Linguistics, 8:842-866.

Ruslan Salakhutdinov and Geoffrey E. Hinton. 2009. Replicated softmax: an undirected topic model. In Advances in Neural Information Processing Systems 22: 23rd Annual Conference on Neural Information Processing Systems 2009, pages 1607-1614. Curran Associates, Inc.

Akash Srivastava and Charles Sutton. 2017. Autoencoding variational inference for topic models. In 5 th International Conference on Learning Representations, ICLR 2017, Toulon, France, April 24-26, 2017, Conference Track Proceedings.

Silvia Terragni, Elisabetta Fersini, Bruno Giovanni Galuzzi, Pietro Tropeano, and Antonio Candelieri. 2021a. OCTIS: Comparing and optimizing topic models is simple! In Proceedings of the 16th Conference of the European Chapter of the Association for Computational Linguistics: System Demonstrations, pages 263-270, Online. Association for Computational Linguistics.

Silvia Terragni, Elisabetta Fersini, and Enza Messina. 2020a. Constrained relational topic models. Information Sciences, 512:581-594.

Silvia Terragni, Elisabetta Fersini, and Enza Messina. 2021b. Word embedding-based topic similarity measures. In Natural Language Processing and Information Systems - 26th International Conference on Applications of Natural Language to Information Systems, NLDB 2021. Springer.

Silvia Terragni, Debora Nozza, Elisabetta Fersini, and Messina Enza. 2020b. Which matters most? comparing the impact of concept and document relationships in topic models. In Proceedings of the First Workshop on Insights from Negative Results in NLP, pages 32-40.
William Webber, Alistair Moffat, and Justin Zobel. 2010. A similarity measure for indefinite rankings. ACM Transactions on Information Systems (TOIS), 28(4):1-38.

Adina Williams, Nikita Nangia, and Samuel Bowman. 2018. A broad-coverage challenge corpus for sentence understanding through inference. In Proceedings of the 2018 Conference of the North American Chapter of the Association for Computational Linguistics: Human Language Technologies, Volume 1 (Long Papers), pages 1112-1122, New Orleans, Louisiana. Association for Computational Linguistics.

Guangxu Xun, Yaliang Li, Wayne Xin Zhao, Jing Gao, and Aidong Zhang. 2017. A correlated topic model using word embeddings. In Proceedings of the Twenty-Sixth International Joint Conference on Artificial Intelligence, IJCAI 2017, pages 4207-4213. ijcai.org.

Yi Yang, Doug Downey, and Jordan L. Boyd-Graber. 2015. Efficient methods for incorporating knowledge into topic models. In Proceedings of the 2015 Conference on Empirical Methods in Natural Language Processing, EMNLP 2015, pages 308-317. The Association for Computational Linguistics.

He Zhao, Lan Du, Wray Buntine, and Gang Liu. 2017. Metalda: A topic model that efficiently incorporates meta information. In 2017 IEEE International Conference on Data Mining (ICDM), pages 635-644. IEEE.

He Zhao, Dinh Phung, Viet Huynh, Yuan Jin, Lan Du, and Wray Buntine. 2021. Topic modelling meets deep neural networks: A survey. arXiv preprint arXiv:2103.00498.

\section{A Datasets}

We pre-processed 20NewsGroup and Wiki20K. We removed punctuation, digits, and nltk's English stop-words. Following other researchers, we selected 2,000 as the maximum number of words for the BoW, and thus we kept only the 2,000 most frequent words in the documents. The other datasets come already pre-processed (reference links are in the paper) and thus we take them as is.

\section{B Models and Baselines}

\section{B.1 ProdLDA}

We use the implementation made available by Carrow (2018) since it is the most recent and with the most updated packages (e.g., one of the latest versions of PyTorch). We run 100 epochs of the model. We use ADAM optimizer. The inference network is composed of a single hidden layer and 100-dimension of softplus units. The priors over 
the topic and document distributions are learnable parameters. Momentum is set to 0.99 , the learning rate is set to 0.002 , and we apply $20 \%$ of drop-out to the hidden document representation. The batch size is equal to 200. More details related to the architecture can be found in the original work (Srivastava and Sutton, 2017).

\section{B.2 Combined TM}

The model and the hyper-parameters are the same used for ProdLDA with the difference that we also use SBERT features in combination with the BoW: we take the SBERT English embeddings, apply a (learnable) function/dense layer $R^{1024} \rightarrow R^{|V|}$ and concatenate the representation to the BoW. We run 100 epochs of the model. We use ADAM optimizer.

\section{B.3 LDA}

We use Gensim's ${ }^{9}$ implementation of this model. The hyper-parameters $\alpha$ and $\beta$, controlling the document-topic and word-topic distribution respectively, are estimated from the data during training.

\section{B.4 ETM}

We use the implementation available at https: //github.com/adjidieng/ETM with default hyperparameters.

\section{B.5 Meta-LDA}

We use the authors' implementation available at https://github.com/ethanhezhao/MetaLDA. As suggested, we use the Glove embeddings to initialize the models. We used the 50-dimensional embeddings from https://nlp.stanford.edu/ projects/glove/. The parameters $\alpha$ and $\beta$ have been set to 0.1 and 0.01 respectively.

\section{B.6 Neural Variational Document Model (NVDM)}

We use the implementation available at https: //github.com/ysmiao/nvdm with default hyperparameters, but using two alternating epochs for encoder and decoder.

\section{Computing Infrastructure}

We ran the experiments on two common laptops, equipped with a GeForce GTX 1050: models can be easily run with basic infrastructure (having a GPU is better than just using CPU, but the experiment can also be replicated with CPU). Both lap-

\footnotetext{
${ }^{9}$ https: / / radimrehurek.com/gensim/ models/ldamodel.html
}

tops have 16GB of RAM. CUDA version for the experiments was 10.0.

\section{C.1 Runtime}

What influences the computational time the most is the number of words in the vocabulary. Table 5 shows the runtime for one epoch of both our Combined TM (CTM) and ProdLDA (PDLDA) for 25 and 50 topics on Google News and 20Newsgroups datasets with the GeForce GTX 1050. ProdLDA is faster than our Combined TM. This is due to the added representation. However, we believe that these numbers are quite similar and make our model easy to use, even with common hardware.

\begin{tabular}{c|cc|cc}
\hline & \multicolumn{2}{|c|}{ GNEWS } & \multicolumn{2}{c}{ 20NG } \\
\hline & 50 topics & 100 topics & 50 topics & 100 topics \\
\hline CTM & $2.1 \mathrm{~s}$ & $2.2 \mathrm{~s}$ & $1.2 \mathrm{~s}$ & $1.2 \mathrm{~s}$ \\
PLDA & $1.5 \mathrm{~s}$ & $1.5 \mathrm{~s}$ & $0.8 \mathrm{~s}$ & $0.9 \mathrm{~s}$ \\
\hline
\end{tabular}

Table 5: Time to complete one epoch on Google News and 20 Newsgroups datasets with 25 and 50 topics. 